\title{
A comparison of postoperative quality of life and dysfunction after Billroth I and Roux-en-Y reconstruction following distal gastrectomy for gastric cancer: results from a multi-institutional RCT
}

\author{
Shuji Takiguchi $\cdot$ Kazuyoshi Yamamoto $\cdot$ Motohiro Hirao $\cdot$ Hiroshi Imamura \\ Junya Fujita • Masahiko Yano • Kenji Kobayashi • Yutaka Kimura • Yukinori Kurokawa • \\ Masaki Mori • Yuichiro Doki • Osaka University Clinical Research Group for Gastroenterological Study \\ Received: 10 May 2011 / Accepted: 11 September 2011/Published online: 13 October 2011 \\ (c) The International Gastric Cancer Association and The Japanese Gastric Cancer Association 2011
}

\begin{abstract}
Background Both Billroth I (B-I) and Roux-en-Y (R-Y) reconstructions are commonly performed as standard procedures, but it has yet to be determined which reconstruction is better for patients. A randomized prospective phase II trial with body weight loss at 1 year after surgery as a primary endpoint was performed to address this issue. The current report delivers data on the quality of life and degree of postoperative dysfunction, which were the secondary endpoints of this study.
\end{abstract}

S. Takiguchi $(\bowtie) \cdot$ K. Yamamoto $\cdot$ Y. Kurokawa $\cdot$ M. Mori

Y. Doki

Department of Gastroenterological Surgery,

Osaka University Graduate School of Medicine,

2-2 Yamadaoka, Suita, Osaka 565-0876, Japan

e-mail: stakigichi@gesurg.med.osaka-u.ac.jp

M. Hirao

Department of Surgery, Osaka National Hospital, Osaka, Japan

H. Imamura

Department of Surgery, Sakai Municipal Hospital, Osaka, Japan

J. Fujita

Department of Surgery, Toyonaka Municipal Hospital,

Osaka, Japan

M. Yano

Department of Surgery, Osaka Medical Center for Cancer and

Cardiovascular Diseases, Osaka, Japan

K. Kobayashi

Department of Surgery, Kinki Chuou Hospital, Itami, Japan

Y. Kimura

Department of Surgery, NTT West Japan Hospital, Osaka, Japan
Methods Gastric cancer patients who underwent distal gastrectomy were intraoperatively randomized to B-I or R-Y. Postsurgical QOL was evaluated using the EORTC QLQ-C30 and DAUGS 20.

Results Between August 2005 and December 2008, 332 patients were enrolled in a randomized trial comparing B-I versus R-Y. A mail survey questionnaire sent to 327 patients was completed by $268(86.2 \%)$ of them. EORTC QLQ-C30 scores were as follows: global health status was similar in each group (B-I $73.5 \pm 18.8, \mathrm{R}-\mathrm{Y} 73.2 \pm 20.2$, $p=0.87)$. Scores of five functional scales were also similar. Only the dyspnea symptom scale showed superior results for $\mathrm{R}-\mathrm{Y}$ than for $\mathrm{B}-\mathrm{I}$ (B-I $13.6 \pm 17.9$, R-Y $8.6 \pm 16.3, p=0.02)$. With respect to DAUGS 20 , the total score did not differ significantly between the R-Y and B-I groups (24.8 vs. 23.6, $p=0.41)$. Only reflux symptoms were significantly worse for B-I than for R-Y $(0.7 \pm 0.6$ vs. $0.5 \pm 0.6, p=0.01)$.

Conclusions The B-I and R-Y techniques were generally equivalent in terms of postoperative QOL and dysfunction. Both procedures seem acceptable as standard reconstructions after distal gastrectomy with regard to postoperative QOL and dysfunction.

Keywords Distal gastrectomy · Roux-en-Y · Billroth I · QOL · Randomized trial

\section{Introduction}

Both Billroth I (B-I) and Roux-en-Y (R-Y) anastomoses have been performed as standard procedures after distal gastrectomy [1]. B-I was once commonly performed because this procedure was simple and the foods passed physiologically [2]. R-Y reconstruction was chosen to 
prevent postoperative alkaline reflux gastritis and esophagitis of the remnant stomach after distal gastrectomy [3-5]. In addition to these problems, some surgeons reported postoperative carcinogenesis of the remnant stomach [6-8]. In contrast, R-Y stasis syndrome, which occurs occasionally during the early postoperative period, is one of the major complications of R-Y reconstruction [9-11]. Most surgeons choose a reconstruction procedure according to personal preferences or degree of experience. It is difficult to select the reconstruction procedure scientifically because few studies have directly compared the B-I and R-Y techniques.

We performed a randomized prospective multicenter trial comparing B-I and R-Y reconstruction. The primary endpoint was a comparison of body weight loss 1 year after surgery. Postoperative quality of life (QOL) was one of the secondary endpoints of the study.

QOL evaluation using questionnaire surveys was once considered to be unreliable because of their subjective nature. However, questionnaires have since been developed and validated as important tools for comprehensively assessing physical conditions. They are now considered to be reliable measurements for evaluating surgical outcomes, especially in randomized clinical trials.

This study is the first to use a questionnaire survey to evaluate QOL and dysfunction following B-I and R-Y reconstructions after distal gastrectomy.

\section{Methods}

\section{Study design}

This prospective trial was initiated in August 2005. We conducted a multicenter randomized phase II study that was approved by the institutional review boards of all participating hospitals and was conducted in accordance with the Declaration of Helsinki. Our hypothesis was that $\mathrm{R}-\mathrm{Y}$ reconstruction would result in lower postoperative body weight loss than the B-I technique while maintaining similar surgical results. The primary endpoint was postoperative body weight loss, and secondary endpoints were surgical morbidity and postoperative QOL.

\section{Patients}

After completion of the informed consent process, patients were included in the study if they met the following eligibility criteria: histologically proven gastric cancer, a lack of non-curative surgical factors except for positive lavage cytology, age between 20 and 90 years, an Eastern Cooperative Oncology Group (ECOG) performance status of $0-1$, no prior chemotherapy or radiation therapy, and no history of gastrectomy or other malignancy (except carcinoma in situ of uterus cervical cancer and focal cancer in adenoma of colorectal cancer) during the past 5 years. All patients gave written informed consent before undergoing randomization. Exclusion criteria included: history of laparotomy (except appendectomy and laparoscopic cholecystectomy), interstitial pneumonia or pulmonary fibrosis, severe heart disease, liver cirrhosis or active hepatitis, chronic renal failure, severe diabetes ( $\mathrm{HbA} 1 \mathrm{c} \geq 9.0 \%$ ), and severe reflux esophagitis. After the surgeon confirmed the above eligibility and exclusion criteria immediately following the initial laparotomy, patients were intraoperatively randomized to either the B-I group or the R-Y group. Randomization was performed by the minimization method according to BMI and institutional preferences.

In our surgical study group, the Osaka University Clinical Research Group for Gastroenterological Study, the standard reconstructive method following distal gastrectomy has been the BI reconstruction because of the physiological advantage of allowing food to pass through the duodenum and the surgical simplicity of the BI reconstructive method in comparison with the RY method. It has been reported that the rate of body weight loss at 1 postoperative year was $10-15 \%$ following BI operations [12]. In this study we hypothesized that relative to the BI operation, the RY operation may decrease body weight loss at 1 year after surgery by $5 \%$.

The sample size was determined to provide $80 \%$ power to detect an effect size of $5 \%$ using a one-sided alpha error of $5 \%$ under the normal distribution with a standard deviation of 0.1 in both groups. The primary endpoint was evaluated by $t$ test. The planned sample size was 320 patients (160 for each arm), allowing for a 10\% dropout rate at the postoperative 1-year point.

\section{Surgical treatment}

In both groups, the surgeon performed standard treatment for gastric cancer according to the Japan classification of gastric carcinoma [13]. As a result of this study's design as a multicenter trial, a variety of procedures were employed during reconstructions, including use of mechanical suture devices or hand-sewn techniques, choice of antecolic or retrocolic routes during the R-Y approach, and laparoscopic or open procedures. There were no detailed regulations concerning each reconstruction procedure so as to provide patients with the highest quality of care based on the specific institution in which they were hospitalized. The only requirement was an R-Y limb length of $30 \mathrm{~cm}$, because this length could affect postoperative nutrition and R-Y stasis.

Patients were enrolled from 18 hospitals belonging to the Osaka University Clinical Research Group for Gastroenterological Surgery. All operations were performed or 
supervised by senior surgeons who were members of the Japaneses Gastric Cancer Association. Patients were followed up every 3 or 6 months until 5 postoperative years. Adjuvant therapy was not specified in the protocol.

\section{Assessment of QOL}

The European Organization for Research and Treatment of Cancer (EORTC) Core Quality of Life Questionnaire (QLQ-C30) (Japanese version) is a 30-item cancer-specific integrated system for assessing the health-related QOL of cancer patients [14-16]. The questionnaire comprises five scales related to function (physical role, cognitive, emotional, and social), three symptom scales (fatigue, pain, and nausea and vomiting), a global health and QOL scale, and single items for the assessment of additional symptoms commonly reported by cancer patients (e.g., dyspnea, appetite loss, sleep disturbance, constipation, and diarrhea), and perceived financial impact of the disease and treatment. All items are scored using 4-point Likert scales. All scales were linearly transformed to a 0 to 100 score, with 100 representing the best global health status or functional status or the worst symptom status.

\section{Assessment of postoperative dysfunction}

The Dysfunction After Upper Gastrointestinal Surgery for Cancer (DAUGS 20) scoring system was to assess postoperative dysfunction. The DAUGS 20 has previously undergone extensive development and testing [17, 18], and was originally developed for simultaneous use with the EORTC QLQ-C30. The patients rated 20 items related to postoperative gastrointestinal dysfunction according to a scale of 1 (not at all) to 5 (very severe). High scores indicated more severe dysfunction. The 20 items were divided into the following 7 categories: (1) diarrhea or soft feces, (2) pain, (3) dumping-like symptoms, (4) food passage dysfunction, (5) nausea and vomiting, (6) decreased physical activity, and (7) reflux symptoms.

\section{Questionnaire survey}

A self-administered questionnaire that included the EORTC QLQ-C30 and DAUGS 20 was dispatched by mail 3 months after the last case had been registered. The patients completed the questionnaire and returned it by mail to the clinical study register center. Because this questionnaire survey was not administered by a primary care doctor, bias was minimized.

Statistical analysis

Stastical analysis was performed with the JMP stastical package, version 8 (SAS, Cary, NC, USA). Data are expressed as means \pm SD. Total scores for the EORTC QLQ-C30 and DAUGS 20 were compared between the two groups using the Mann-Whitney test. $p$ values of less than 0.05 were considered significant.

\section{Results}

Questionnaire, compliance, and missing data

A CONSORT flowchart of the trial design is shown in Fig. 1. A total of 332 adult patients (220 men and 112 women) with gastric cancer were enrolled: 163 in the B-I group and 169 in the R-Y group. Five cases were excluded because of errors in which the reconstruction procedure was performed $(n=3)$ or death $(n=2)$. Of the 327 participants, $282(86.2 \%)$ returned the questionnaire sheets. Fourteen cases were excluded from the analysis because of curability $\mathrm{C}$ (definite residual tumor) and recurrence $(n=9)$ and ongoing adjuvant chemotherapy $(n=5)$, which would strongly affect postoperative QOL and dysfuntion. Finally, 268 cases (132 B-I, 136 R-Y) were analyzed for the evaluation of postoperative QOL. The median observation period was 21 months (range 3-34). The clinicopathological characteristics of the 268 patients are summarized in Table 1. No significant differences were observed in age, sex, depth of tumor invasion, or stage of gastric cancer. The rates of distant and lymphatic metastasis were also similar. The laparoscopic approach was selected in 29 of 163 patients who underwent B-I reconstruction and 33 of 169 patients who were treated by R-Y. Blood loss did not differ between the two groups. The operative time in the R-Y group was significantly longer than in the B-I group (214 vs. $180 \mathrm{~min}$, respectively, $p<0.0001$ ).

\section{EORTC QLQ-C30}

The results of global health status and functional scales of EORTC QLQ-C30 are shown in Fig. 2. The mean scores for global health status were very similar in both groups (B-I $73.5 \pm 21.3$, R-Y $73.2 \pm 20.2, p=0.87$ ). As for the functional scales, B-I was not significantly superior to R-Y on only the cognitive scale (B-I $80.3 \pm 18.1$, R-Y $75.7 \pm 21.3, p=0.06$ ). There were no significant differences between the two groups on the other four functional scales (physical, role, emotional, and social functioning). The results of symptom scales of EORTC QLQ-C30 are shown in Fig. 3. Regarding symptom scales, B-I was significantly inferior to R-Y on the dyspnea scale (B-I $13.6 \pm 17.9$, R-Y $8.6 \pm 16.3, p=0.02$ ). There were no significant differences on the other eight symptom scales (fatigue, nausea and vomiting, pain, insomnia, appetite loss, constipation, diarrhea, financial difficulties). 
Fig. 1 Consort flow chart

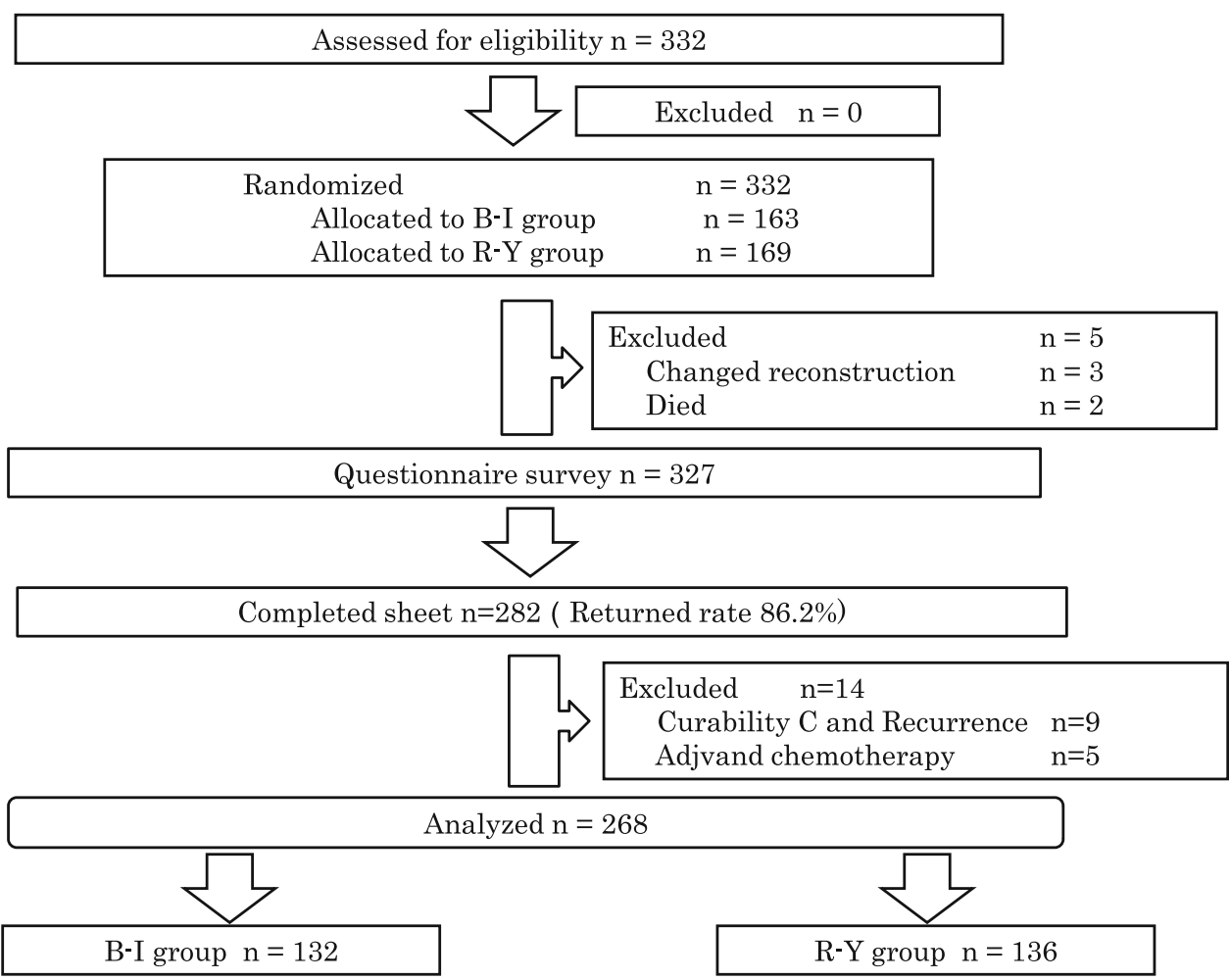

Table 1 Characteristics and operative results of patients who underwent distal gastrectomy and answered the questionnaire survey

\begin{tabular}{llll}
\hline & B-I group $(n=132)$ & R-Y group $(n=136)$ & $p$ \\
\hline Sex (male/female) & $105 / 58$ & $113 / 53$ & $0.48^{*}$ \\
Age & $64.5 \pm 9.8$ & $64.1 \pm 10.5$ & $0.68^{\dagger}$ \\
Height (cm) & $161.3 \pm 8.3$ & $161.1 \pm 9.7$ & $0.89^{\dagger}$ \\
Weight (kg) & $58.3 \pm 9.7$ & $59.5 \pm 11.3$ & $0.29^{\dagger}$ \\
Macroscopic appearance (0/1/2/3/5) & $98 / 5 / 17 / 9 / 3$ & $100 / 8 / 13 / 14 / 1$ & $0.50^{* *}$ \\
Location (L/M) & $92 / 40$ & $91 / 45$ & $0.62^{*}$ \\
Tumor size (cm) & $2.9 \pm 1.7$ & $2.9 \pm 1.5$ & $136 / 33$ \\
Approach (open/laparoscopic) & $134 / 29$ & $59 / 106 / 1$ & $0.93^{*}$ \\
Dissection level (D1+/D2/D3) & $58 / 105 / 0$ & $214 \pm 44$ & $0.68^{*}$ \\
Operation time (min) & $180 \pm 48$ & $203 \pm 153$ & $0.61^{*}$ \\
Blood loss (ml) & $210 \pm 230$ & $45 / 57 / 17 / 13 / 4$ & $104 / 32$ \\
m/sm/mp/ss/se & $48 / 54 / 15 / 11 / 4$ & $90001^{\dagger}$ \\
pN (-/+) & $107 / 25$ & $0.78^{\dagger}$ \\
pStage (IA/IB/II/IIIA/IIIB/IV) & $91 / 24 / 15 / 2 / 0 / 0$ & $0.98^{* *}$ \\
\hline
\end{tabular}

Clinical findings and staging classifications are described according to the Japanese Classification of Gastric Carcinoma

$* \chi^{2}$ test

** Mann-Whitney $U$ test

$\dagger$ Wilcoxon rank sum test

\section{DAUGS 20 scoring system}

The results of the DAUGS 20 score are shown in Fig. 4. The total score of the DAUGS 20 was very similar in both groups (B-I $24.8 \pm 11.6, \mathrm{R}-\mathrm{Y} 23.6 \pm 11.4, p=0.41$ ). Subclass analysis showed that B-I was significantly worse in terms of reflux symptoms (B-I $0.7 \pm 0.6, \quad \mathrm{R}-\mathrm{Y}$ $0.5 \pm 0.6, p=0.01)$. There were no significant differences between the two groups in terms of the other six subclasses: diarrhea or soft feces (B-I $2.1 \pm 1.3$, R-Y $2.0 \pm 1.2$, $p=0.7$ ), pain (B-I $1.1 \pm 0.9$, R-Y $1.2 \pm 0.9, p=0.64$ ), dumping-like syndrome (B-I $1.8 \pm 1.0, \mathrm{R}-\mathrm{Y} 1.8 \pm 1.0$, 


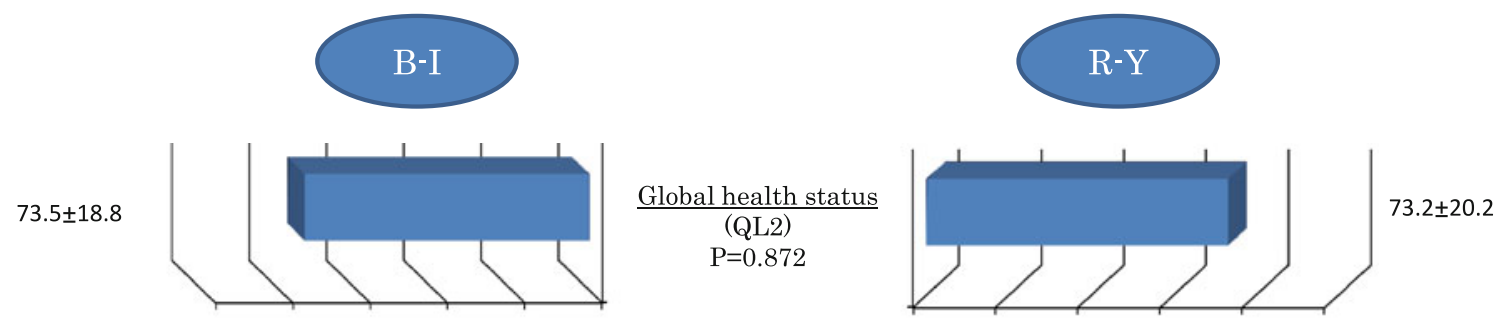

Functional scales

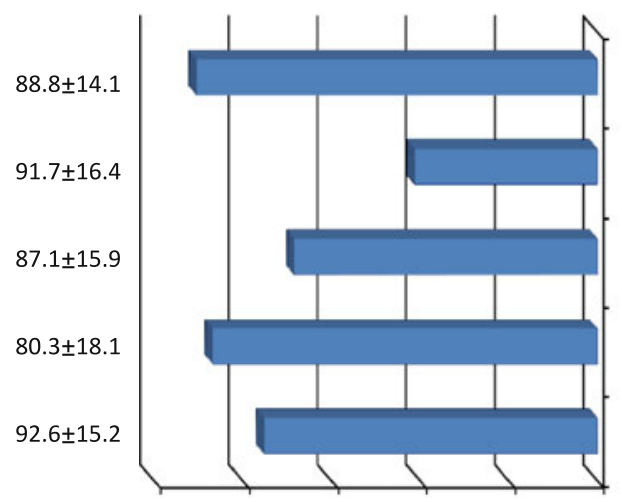

Physical functioning $\mathrm{P}=0.89$

Role functioning $\mathrm{P}=0.93$

Emotional functioning $\mathrm{P}=0.35$

Cognitive functioning $\mathrm{P}=0.06$

Social functioning $\mathrm{P}=0.61$

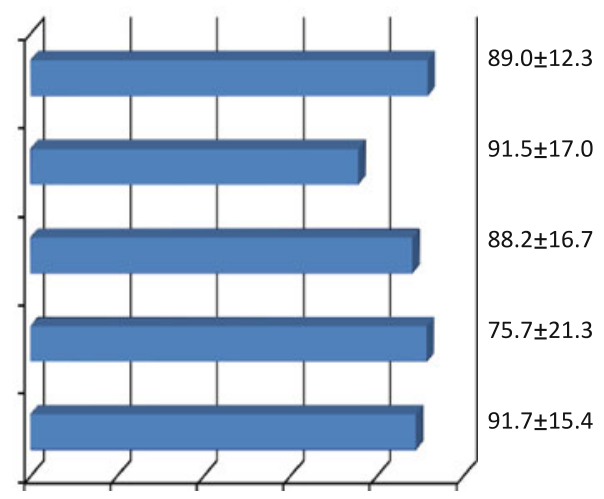

Wilcoxon rank sum test

Fig. 2 The mean scores for global health status were very similar in both groups (B-I $73.5 \pm 21.3$, R-Y 73.2 $\pm 20.2, p=0.87$ ). As for the functional scales, B-I was nonsignificantly superior to R-Y on only the cognitive scale (B-I $80.3 \pm 18.1, \mathrm{R}-\mathrm{Y} 75.7 \pm 21.3, p=0.06$ )

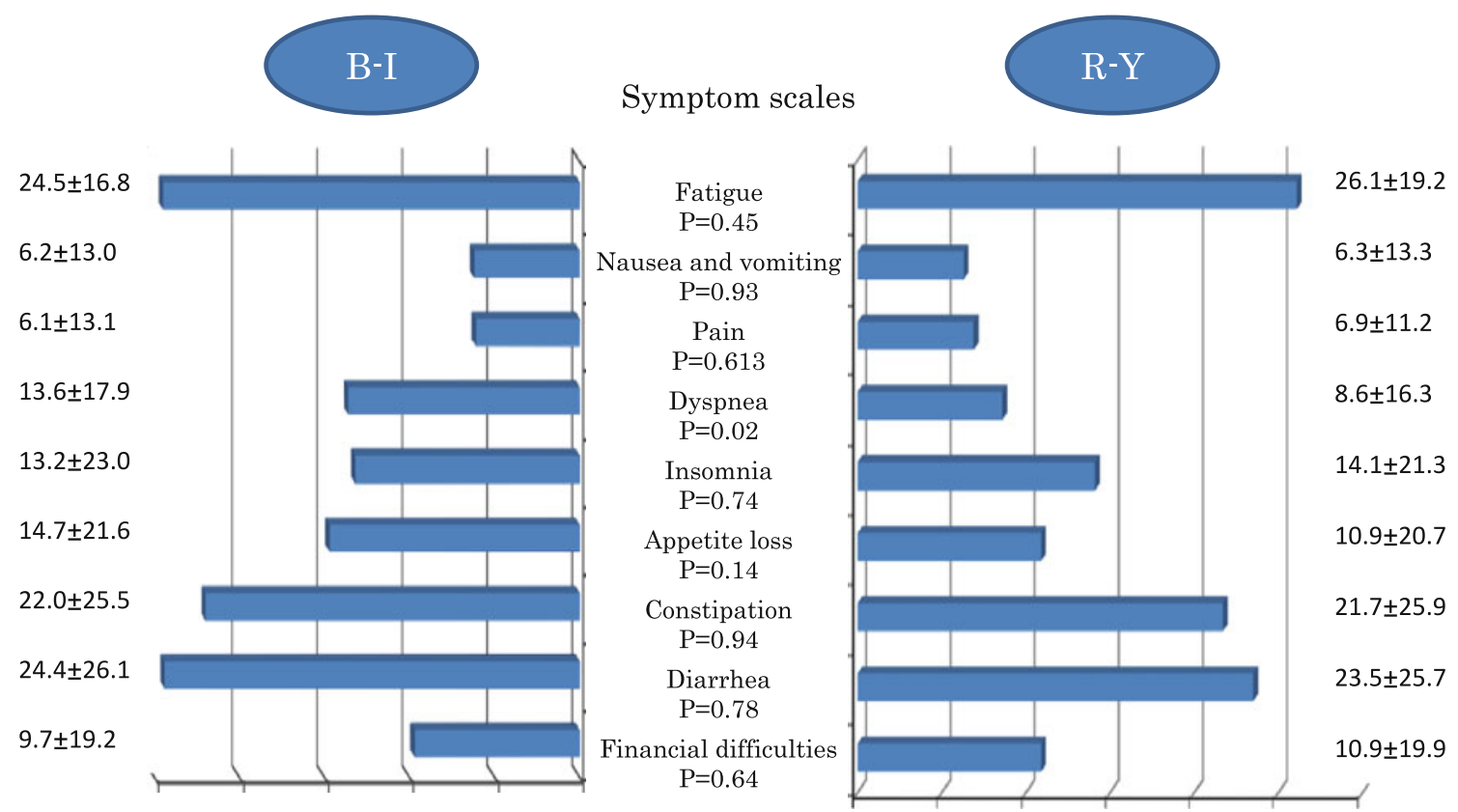

Wilcoxon rank sum test

Fig. 3 B-I was significantly inferior to R-Y on the dyspnea scale (B-I $13.6 \pm 17.9, \mathrm{R}-\mathrm{Y} 8.6 \pm 16.3, p=0.02)$. There were no significant differences on the other eight symptom scales (fatigue, nausea and vomiting, pain, insomnia, appetite loss, constipation, diarrhea, financial difficulties) 


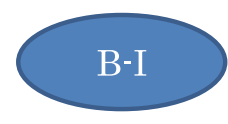

Total score: $24.8 \pm 11.6$
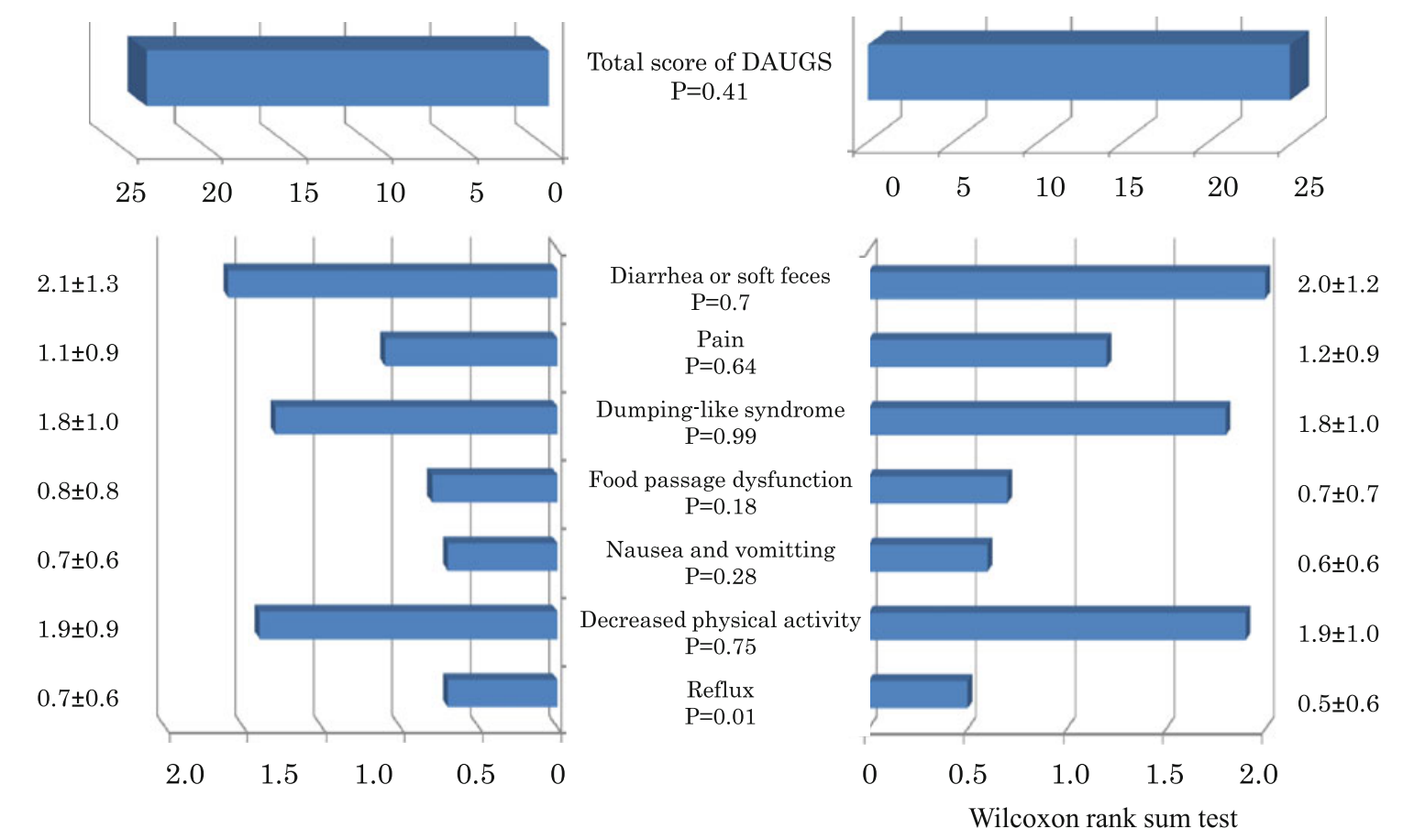

$\mathrm{R}-\mathrm{Y}$

Total score: $23.6 \pm 11.4$

Fig. 4 The total score of the DAUGS 20 was very similar in both groups (B-I $24.8 \pm 11.6$, R-Y $23.6 \pm 11.4, p=0.41$ ). Subclass analysis showed that B-I was significantly worse in terms of reflux symptoms (B-I $0.7 \pm 0.6, \mathrm{R}-\mathrm{Y} 0.5 \pm 0.6, p=0.01$ )

$p=0.99$ ), food passage dysfunction (B-I $0.8 \pm 0.8$, R-Y $0.7 \pm 0.7, p=0.18$ ), nausea and vomiting (B-I $0.7 \pm 0.6$, $\mathrm{R}-\mathrm{Y} 0.6 \pm 0.6, \mathrm{P}=0.28)$, and decreased physical activity (B-I $1.9 \pm 0.9, \mathrm{R}-\mathrm{Y} 1.9 \pm 1.0, p=0.75$ ).

Comparison of survey scores every 6 months

The global health status scores and total DAUGS 20 scores were summarized every 6 months (Fig. 5). There were significant differences in total DAUGS 20 scores during the first 6 months (B-I $22.8 \pm 13.7$, R-Y $32.4 \pm 8.9$, $p=0.04)$. There was no significant difference in global health status and total DAUGS 20 scores at other periods between the B-I group and the R-Y group.

\section{Discussion}

This prospective randomized trial showed no significant differences between the B-I and R-Y groups in terms of postoperative QOL and dysfunction, as evaluated by a questionnaire using the EORTC QLQ-C30 and DAUGS 20 scales. In this study, body weight loss at 1 year after surgery, which was the primary endpoint in this study, was
9.1\% for the B-I group and $9.7 \%$ for the R-Y group $(p=0.39)$. Body weight change would be related to the QOL and dysfunction. The results of the questionnaire survey did not contradict the results of body weight loss. This study included a larger number of cases than other randomized clinical trials evaluating postoperative QOL and dysfunction after distal gastrectomy. It was particularly interesting that patients in the two groups evaluated their QOL and dysfunction almost equally despite the significant anatomic differences between the reconstruction procedures.

Prognosis or overall survival has been the most important factor when evaluating cancer treatments. Since cancer is now detected more frequently in its early stages and postoperative prognosis has improved, postoperative QOL and dysfunction have come to be acknowledged as important endpoints in addition to oncologic outcomes and safety issues. For example, Kim et al. [19] reported that laparoscopy-assisted distal gastrectomy was superior to conventional open distal gastrectomy in terms of QOL outcomes 3 months after surgery. Precise evaluation of the effectiveness of minimally invasive surgery is difficult; however, if the oncologic outcome is equal between procedures, QOL findings will be useful in deciding on the 
Fig. 5 There were significant differences in total DAUGS 20 scores during the first 6 months (B-I $22.8 \pm 13.7$, R-Y $32.4 \pm 8.9, p=0.04)$. There was no significant difference in global health status and total DAUGS 20 scores at other periods between the B-I group and the R-Y group
(A) QOL scores; Global health status

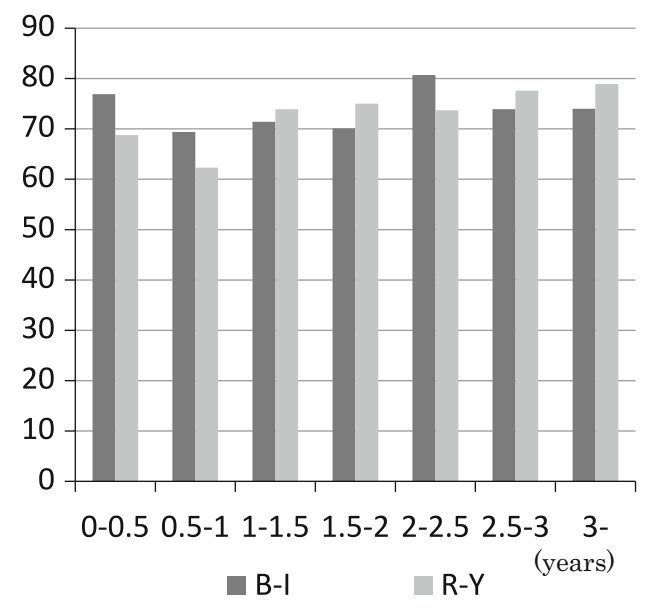

(B) Total DAUGS Scores

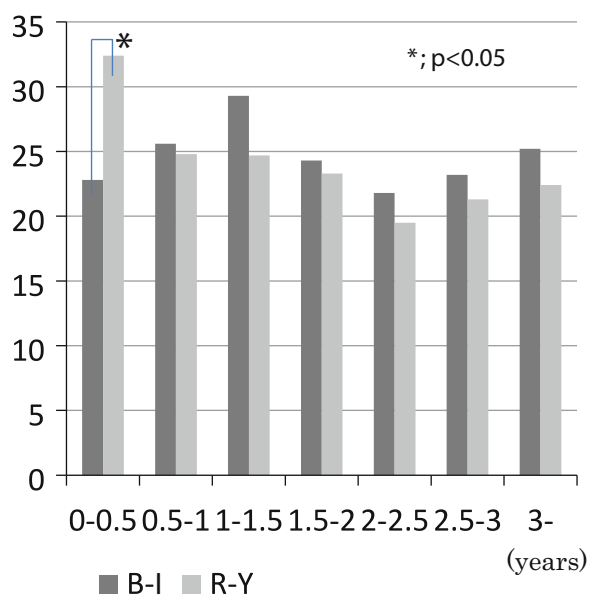

optimal operative approach. The Japanese version of the EORTC QLQ-C30 has been developed and validated. Kobayashi et al. [20] used this version to prospectively compare postoperative health-related QOL among gastrectomy patients and found clear difference among the operative procedures.

The DAUGS 20 scale was designed to objectively assess gastrointestinal dysfunction after surgery for upper gastrointestinal cancer. The scale has already been validated in the field of upper intestinal cancer $[17,18]$. We found no significant difference between R-Y and B-I procedures in terms of overall postoperative dysfunction. However, there were significant differences in total DAUGS 20 scores during the first 6 months (B-I 22.8 \pm 13.7 , R-Y $32.4 \pm 8.9, p=0.04)$. Especially each score of food passage dysfunction and nausea and vomiting tended to be worse in the R-Y group (not significant). This may be weakened to gastrointestinal motility and delayed gastric emptying with R-Y. In this series, the frequency of nausea, vomiting, and discontinuation of food intake were significantly lower in the B-I group than in the R-Y group (3.7 vs. $12.4 \%, p=0.0027 ; 3.1$ vs. $8.9 \%, p=0.022 ; 4.3$ vs. $12.4 \%, p=0.0064$, respectively). Frequency of delayed gastric emptying in the B-I group was lower than in the R-Y group (4.3 vs. 9.5\%, $p=0.057$ ). In general, Roux en $\mathrm{Y}$ stasis occurred within the postoperative 1st month. Minor symptoms could not be detected during the hospital stay, and small amounts of nausea or vomiting might have occurred at home. Our questionnaire survey might detect this small difference between B-I and R-Y related gastrointestinal motility during the first 6 months.

The EORTC QLQ-C30 showed significant differences only in dyspnea. This symptom seemed to be physiologically unrelated to postoperative complications. Patients who received B-I gastrectomy sometimes complained of heartburn. This score seemed to be affected by esophagitis caused by bile and gastric juice reflux. In the analysis of partial items of the DAUGS, reflux symptoms also obviously appeared in the B-I group. If this limitation can be overcome, we can feel confident in continuing to perform B-I reconstructions. While Shibata reported that semifundoplication following B-I reconstruction prevented this difficulty, further surgical intervention following gastrectomy is less than ideal [21].

The questionnaire survey in the current study was performed only once for each patient and at varying time points after surgery, since co-investigators in this multiinstitutional study did not agree to perform the survey several times and at regular intervals. Such a design would have delivered more convincing data, but would have been too much of a burden for the co-investigators. An alternative design would have been to perform all the surveillances at a fixed time point, such as at 1 year postoperatively. However, it was not possible to decide on the optimal time point for performing the surveillance at the time this study was designed. It has now become clear how the scores vary at different time points, and further study to confirm the differences between B-I and R-Y can now be designed and proposed.

In general, from the point of view of the surgeon, B-I reconstruction is considered to be simple and relatively easy. For the patient, nutritional and hormonal advantages might exist in this physiological route. It is easier to treat common bile duct stones using a gastrointestinal fiberscope after B-I reconstruction. In contrast, the advantage of $\mathrm{R}-\mathrm{Y}$ reconstruction is thought to be less anastomotic leakage and infrequent reflux esophagitis and gastritis. However, disadvantages include a more complicated surgical procedure as well as delayed gastric emptying, so-called Rouxen-Y stasis. All surgeons recognize these issues, and their decisions on which approach to use are based on individual experience. Clinical randomized trials are very important 
in providing surgeons with information to facilitate their decision-making. Ishikawa et al. [12] conducted a randomized trial and showed that B-I reconstruction was superior to R-Y in terms of shorter postoperative hospital stay. At the time it was carried out, this study was the first and most important trial comparing B-I and R-Y reconstructions, and many surgeons have referred to its results as clinical evidence. Our study should also be useful in assisting surgeons with deciding between the two procedures.

In summary, this questionnaire survey using the EORTC QLQ-C30 and DAUGS 20 scales revealed that the B-I and $\mathrm{R}-\mathrm{Y}$ reconstruction approaches were nearly equal in terms of postoperative QOL and dysfunction. It is noteworthy, however, that B-1 was significantly better regarding the total DAUGS 20 score during the first 6 months after surgery. The current study revealed differences in QOL and postoperative dysfunction scores between the two modes of reconstruction at various time points. More refined prospective trials with improved designs based on these results have to be proposed.

Acknowledgments The authors thank Dr. Tomoyuki Sugimoto at Osaka University for help with the statistical analysis. Participating institutions and chief participants: Sakai Municipal Hospital (H. Furukawa, H. Imamura), Osaka University (Y. Doki, S. Takiguchi), Osaka National Hospital (T. Tsujinaka, K. Fujitani), Toyonaka Municipal Hospital (J. Fujita, Kawanishi), Osaka Medical Center for Cancer and Cardiovascular Diseases (M. Yano, I. Miyashiro), Kinki Central Hospital of the Mutual Aid Association of Public School Teachers (K. Kobayashi), NTT West Osaka Hospital (Y. Kimura), Yao Municipal Hospital (Y. Fukushima, H. Matsuyama), Hyogo Prefectural Nishinomiya Hospital (H. Yano, H. Taniguchi), Kansai Rosai Hospital (S. Tamura, H. Miki), Ikeda Municipal Hospital (K. Shibata, T. Hirao), Belland General Hospital (K. Demura), SEMPOS Seamen's Insurance hospital (Y. Tsukahara), Saiseikai Senri Hospital (H. Fukunaga), Nishinomiya Municipal Hospital (H. Oka), Suita Municipal Hospital (C. Ebisui, K. Okada), Itami Municipal Hospital (M. Hiratsuka), and Mino Municipal Hospital (S. Iijima, Y. Makari).

Conflict of interest None of the authors have financial or personal conflicts of interest to disclose.

\section{References}

1. Yoshino K. History of gastric cancer surgery. J Jpn Surg Soc. 2000;101:855-60.

2. Weil PH, Buchberger R. From Billroth to PCV: a century of gastric surgery. World J Surg. 1999;23:736-42.

3. Osugi H, Fukuhara K, Tagada N, et al. Reconstructive procedure after distal gastrectomy to prevent remnant gastritis. Hepatogastroenterology. 2004;51:1215-8.
4. Kauer WK, Peters JH, DeMeester TR, et al. Composition and concentration of bile acids reflux into the esophagus of patients with gastroesophageal reflux disease. Surgery. 1997;122:874-81.

5. Svensson JO. Duodenogastric reflux after gastric surgery. Scand J Gastroenterol. 1983;18:729-34.

6. Fein M, Peters JH, Chandrasoma P, et al. Duodenoesophageal reflux induces esophageal adenocarcinoma without exogenous carcinogen. J Gastrointest Surg. 1998;2:260-8.

7. Taylor PR, Mason RC, Filipe MI, et al. Gastric carcinogenesis in the rat induced by duodenogastric reflux without carcinogens, morphology, mucin histochemistry, polyamine metabolism, and labeling index. Gut. 1991;32:1447-54.

8. Sato T, Miwa K, Sahara H. The sequential model of Barrett's esophagus and adenocarcinoma induced by duodeno-esophageal reflux without exogenous carcinogens. Anticancer Res. 2002;22: $39-44$.

9. Mackman S, Lemmer KE, Morrissey JF. Postoperative reflux alkali gastritis and esophagitis. Am J Surg. 1971;121:694-7.

10. Gillison EW, Kusakari K, Bombeck CT, et al. The importance of bile in reflux oesophagitis and the success in its prevention by surgical means. Br J Surg. 1972;59:794-8.

11. Mathias JR, Fernandez A, Sninsky CA, et al. Nausea, vomiting, and abdominal pain after Roux-en-Y anastomosis: motility of the jejuna limb. Gastroenterology. 1985;88:101-7.

12. Ishikawa M, Kitayama J, Kaizaki S, et al. Prospective randomized trial comparing Billroth-I and Roux-en-Y procedures after distal gastrectomy for gastric cancer. World J Surg. 2005;29:1415-20.

13. Japanese Gastric Cancer Association. Japanese classification of gastric carcinoma-2nd English edition. Gastric Cancer. 1998;1: $10-24$.

14. Kobayashi K, Takeda F, Teramukai S, et al. A cross-validation of the European Organization for Research and Treatment of Cancer QLQ-C30 (EORTC QLQ-C30) for Japanese with lung cancer. Eur J Cancer. 1998;34:810-5.

15. Wu C, Chiou J, Ko F, et al. Quality of life after curative gastrectomy for gastric cancer in a randomized controlled trial. Br J Cancer. 2008;98:54-9.

16. Aaronson NK, Ahmedzai S, Bergman B, et al. The European Organization for Research and Treatment of Cancer QLQC30: a quality of life instrument for use in international clinical trials in oncology. J Natl Cancer Inst. 1993;85:365-76.

17. Nakamura M, Kido Y, Yano M, Hosoya Y. Reliability and validity of a new scale to assess postoperative dysfunction after resection of upper gastrointestinal carcinoma. Surg Today. 2005;35:535-42.

18. Nakamura M, Hosoya Y, Yano M, Doki Y, Miyashiro I, Kurashina K, Morooka Y, Kishi K, Lefor AT. Extent of gastric resection impacts patient quality of life: the Dysfunction after Upper Gastrointestinal Surgery for Cancer (DAUGS32) Scoring System. Ann Surg Oncol. 2011;18:314-20.

19. Kim YW, Baik YH, Yun YH, et al. Improved quality of life outcomes after laparoscopy-assisted distal gastrectomy for early gastric cancer: results of a prospective randomized clinical trial. Ann Surg. 2008;248:721-7.

20. Kobayashi D, Kodera Y, Fujiwara M, Koike M, Nakayama G, Nakao A. Assessment of quality of life after gastrectomy using EORTC QLQ-C30 and STO22. World J Surg. 2011;35:357-64.

21. Shibata Y. Effect of semifundoplication with subtotal gastrectomy for prevention of postoperative gastroesophageal reflux. J Am Coll Surg. 2004;198:212-7. 